\title{
Experimental system for testing a superconducting motor at temperatures close to $15 \mathrm{~K}$
}

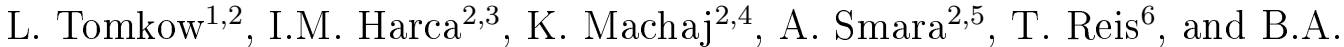 \\ Glowacki $^{2,7}$ \\ ${ }^{1}$ Technology Department, CERN, Esplanade des Particules 1, 1211 Geneva 23, \\ Switzerland \\ ${ }^{2}$ Applied Superconductivity and Cryoscience Group, Department of Materials Science and \\ Metallurgy, University of Cambridge, 27 Charles Babbage Road, Cambridge CB30FS, \\ United Kingdom \\ ${ }^{3}$ Horia Hulubei National Institute for $R \& \mathcal{G} D$ in Physics and Nuclear Engineering \\ (IFIN-HH),Str. Reactorului no. 30, P.O.BOX MG-6, Bucharest-Magurele, Romania \\ ${ }^{4}$ Department of Cryogenics and Aerospace Engineering, Wroclaw University of Science \\ and Technology, Wyb. Wyspianskiego 27, 50-370 Wroclaw, Poland \\ ${ }^{5}$ Theva Dunnschichttechnik GmbH, Rote-Kreuz-Str. 8., 85737 Ismaning, Germany \\ ${ }^{6}$ Oswald Elektromotoren GmbH, Benzstraße 12, 63897 Miltenberg, Germany \\ ${ }^{7}$ Institute of Power Engineering, Ul. Mory 8, 01-330 Warsaw, Poland
}

\begin{abstract}
Integrating superconducting elements in an electric motor can greatly increase its power density. By doing so, lighter and more powerful machines can be produced for applications such as aviation, wind turbines and marine propulsion. Superconducting tapes can be stacked and magnetised to produce powerful trapped-flux magnets. The experimental setup was designed to allow measurements in a low temperature environment, providing tightness for the rotating part.

We report on the design and construction of the setup and results from its first operation. The temperatures achieved during the experimental measurements reached $15 \mathrm{~K}$, close to the actual operational temperatures of a liquid hydrogen cooled motor. The application of hydrogen onboard an aircraft is highly advantageous due to the possibility of its application as both coolant and fuel. Examination of the operation of the trapped-flux magnets at such temperatures is crucial for the understanding of the behavior of fully superconducting motors for future applications.
\end{abstract}

Keywords: Superconductivity, Cryogenics, Electric machines, Rotating 
machines

\section{Introduction}

Power density is a crucial parameter of an electric aircraft [1]. The ratio of power and mass of conventional electric motors is insufficient to economically justify their widespread application in aviation. This property is also important for the applications in wind turbines [2] and marine propulsion [3]. One of the possible methods to address the problem of low power density is the application of stacked superconducting tapes acting as trapped flux magnets [4]. It was observed that such stacks can trap very high magnetic flux, with magnetic induction values up to $17.7 \mathrm{~T}$ [5]. They can perform better than permanent magnets [6], allowing the total weight of the motor using them to be reduced, while increasing the mass power density [7, 8]. Power density can be additionally improved by applying superconducting coils in a stator, forming a fully superconducting motor $[9,10]$. The proposed system could be used in both fully-electric and hybrid planes [11].

The stacks of superconducting tapes have many other advantages. They are relatively cheap, mechanically durable, thermally stable and can be formed into complex shapes [12]. They can carry large currents [13] making their application in fusion reactors and particle accelerators feasible as well [14, 15, 16]. Moreover, such stacks can be produced from otherwise unused materials, such as offcuts from Roebel cables $[17,18]$. In terms of pulsed magnetisation they were found to perform better than superconducting bulks [19]. While the strength of the trapped flux depends on the strength and direction of the magnetising flux, they were found to maintain remarkably repeatable flux in some range of magnetising conditions [20].

The stacks can be tailored to meet specific requirements of a specific application [21, 22]. The tapes in thin stacks can be tilted to increase homogeneity and strength of the trapped flux [23]. Moreover, interlayering different types of tapes can drastically change the properties of the stack. Thermally conducting layers can be added to combat the negative effect of the addition of tapes to the stack on heat removal [24]. Ferromagnetic layers can improve the quality of the trapped flux and decrease demagnetisation [25, 26]. In addition, ferromagnetic inserts were found to improve the magnetic properties of small stacks [27].

The application of trapped-flux magnets in a motor gives rise to several challenges. The behavior of stacks in such environment is complex due to elec- 
tromagnetic and thermal phenomena. Significant differences were observed in operating a stack or a single tape in terms of their characteristics [28]. An experimental measurement system devoted to analyzing the performance of a rotor with integrated trapped-flux magnets is developed and described in this work. By employing the presented system, measurements can be performed at temperatures as low as $15 \mathrm{~K}$, which reflects the expected conditions of an actual motor cooled by hydrogen.

Previous research on a superconducting motor with stacks of tapes working as trapped-flux magnets were performed with setups cooled in liquid nitrogen $[29,30]$. An analysis of possibility of application of cryocooler and gaseous heat transfer medium was to cool an HTS motor down to $40 \mathrm{~K}$ was also performed [31]. The experimental system presented in this paper is cooled with gaseous helium to reach temperatures achievable in an actual motor cooled by liquid hydrogen.

The work describes the design and the initial results on the operation of the motor with the rotor using trapped-field magnets, operating at approximately $15 \mathrm{~K}$. The design of a challenging cryogenic system with moving elements is presented, as well as considerations on the design of the shaft, rotor and stator. Previous applications of gaseous helium for cooling of high temperature superconductors focused on stationary applications, with constant heat leaks and generation, such as DC cables [32]. In this case the dynamic losses are present and the efficient removal of heat is crucial for the stable operation of the motor [33]. Thermal conductivity in the stacks is strongly anisotropic and the majority of heat is removed at the ends connected with the shaft and exposed to helium gas [34, 35].

The major goal of the research planned to be performed using the described system is the analysis of demagnetisation processes in stacks. Demagnetisation occurs as stacks experience time-varying magnetic cross-fields [13, 26] and can lead to changes in the patterns of currents in the stack, decrease of the trapped flux and debut of heat generation [36]. This implies a significant decrease in the performance of the motor itself [37], further exacerbated by the loss of critical currents due to cyclic loads [38]. Experimental measurements and numerical calculations were performed to investigate the demagnetisation process in a motor operating at $77 \mathrm{~K}[39,40]$. The properties of superconducting stacks strongly depend on temperature and quality of the tapes [41, 42]. Additional clarifications were sought while testing the present system. Two methods for stopping demagnetisation are currently researched: re-magnetisation of the stacks employing a pulse of magnetic 
field from the stator [40] and shielding cross-fields with short pieces of superconducting tape placed perpendicularly to the direction of the undesired component [43, 44, 45]. These solutions can be used in complex geometry of the motor [46]. Both can be investigated with the setup described in this paper, in conditions close to actual operation. Beneficial effects can further be achieved by employing mu-metal-based shields [47] which slow down the decay of current in HTS coils [48].

\section{Cryogenic and mechanical design}

\subsection{Cryostat}

The cryostat used in the setup was jointly designed and produced by Applied Superconductivity and Cryoscience Group at University of Cambridge and ICEoxford Ltd. Its simplified diagram is shown in figure 1. The system consists of two separate cold zones. The bottom one is a nitrogen bucket containing a normal-conducting stator. Its second role is maintaining the temperature of the external layer of the tail of the rotor chamber. During operation it is filled with liquid nitrogen under normal pressure and is not actively cooled. The second zone is the rotor chamber, housing the rotor with superconducting stacks and the shaft. It is filled with cold gaseous helium. Both elements are connected to a base plate, placed on a support structure (both visible in figure 2).

A general view of the system is shown in figure 2. On the photography the nitrogen bucket is removed to reveal the tail of the G10 cryostat, part of the vacuum insulation of the rotor chamber. The system is equipped with multiple ports for handling cryogenic liquids and wiring. The rotor chamber is connected to the environment by helium inlet and outlet ports, and wiring port (not shown in the figure). The nitrogen bucket is supplied with nitrogen by a nitrogen inlet port. Two wiring ports are used to power the stator and for measurement read-out. In both cases the outlet ports are equipped with safety valves. The nitrogen bucket is surrounded at the sides and bottom by vacuum insulation. Its top is protected by a thick styrofoam insulation.

The rotor containing the superconducting stacks is placed at the end of the shaft and is located at the bottom of the rotor chamber, as shown in figure 2. The rotor is cooled by cold gaseous helium filling the chamber. The temperature of helium is maintained at the desired level by a Sumitomo CH-110LT cryocooler, placed in the common vacuum vessel with the rotor chamber and connected to a heat exchanger by a long thermal link. The shape of the 


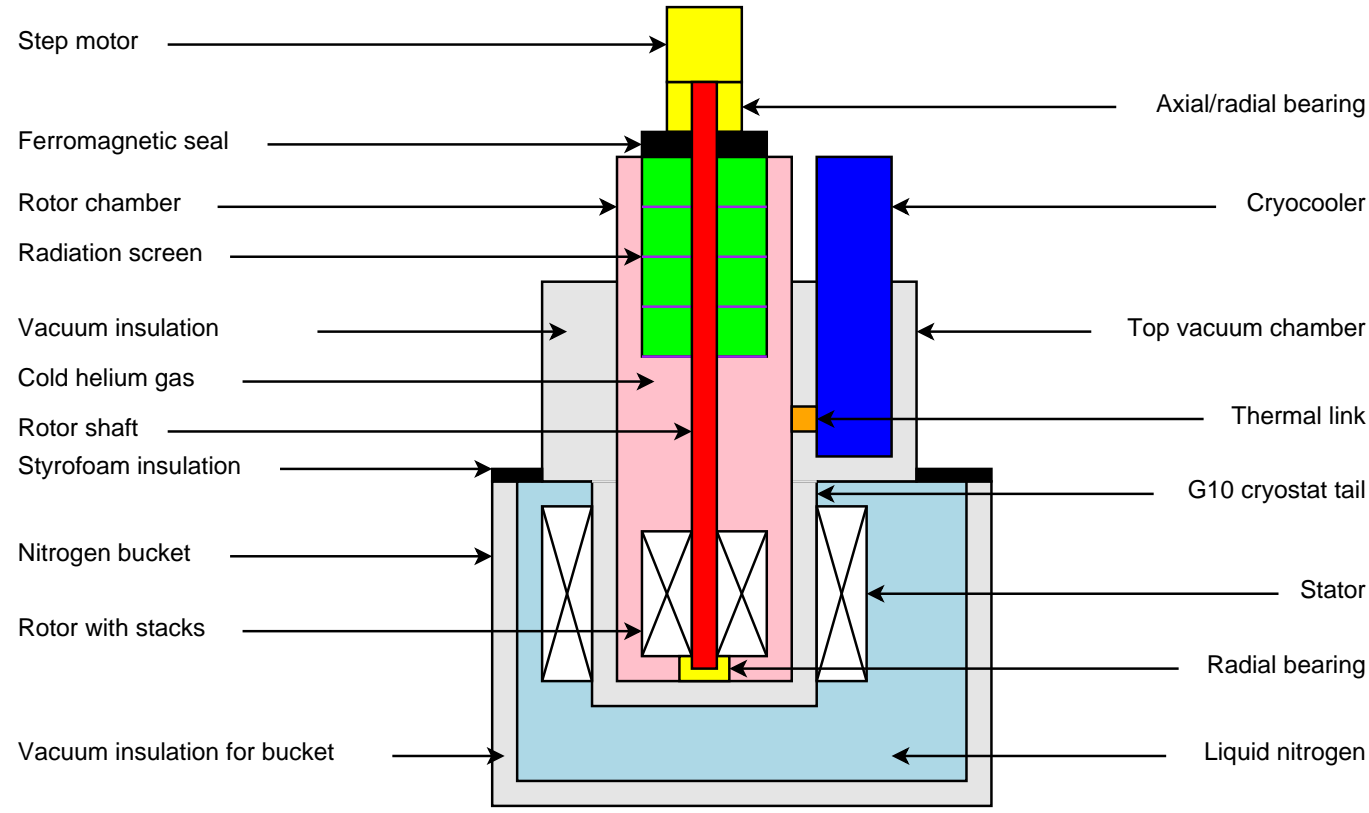

Figure 1: Simple diagram of the present system. Most important components are labeled.

thermal link preserves a stable temperature inside the rotor chamber. The bottom part of the rotor chamber, below the heat exchanger, made of a thin wall of G10, allows to obtain a very small distance between the stator and the rotor. The vacuum insulation of the rotor chamber is connected with the top one. The stator and G10 tail are cooled by liquid nitrogen in the nitrogen bucket. The stator is hanged from the base plate.

\subsection{Rotating assembly}

Figure 4 shows the assembly supporting the rotating parts of the system. The base for the system is flange placed on top of the rotor chamber. Vacuum-tightness of the system is provided by a rotary ferrofluidic seal. Ferromagnetic fluid in the seal is held in place by permanent magnets. Several stages of the fluid stop the propagation of gas. The seal houses also a pair of radial bearings.

Above the seal, a double-sided thrust ball bearing is placed to carry the weight of the shaft and the rotor. The bearing and the shaft are connected by a nut and a locking washer. A coupling is placed at the end of the shaft and connects it to a step motor. Both the bearing and the motor are placed on their own supports on the flange, to minimize the weight carried by the 


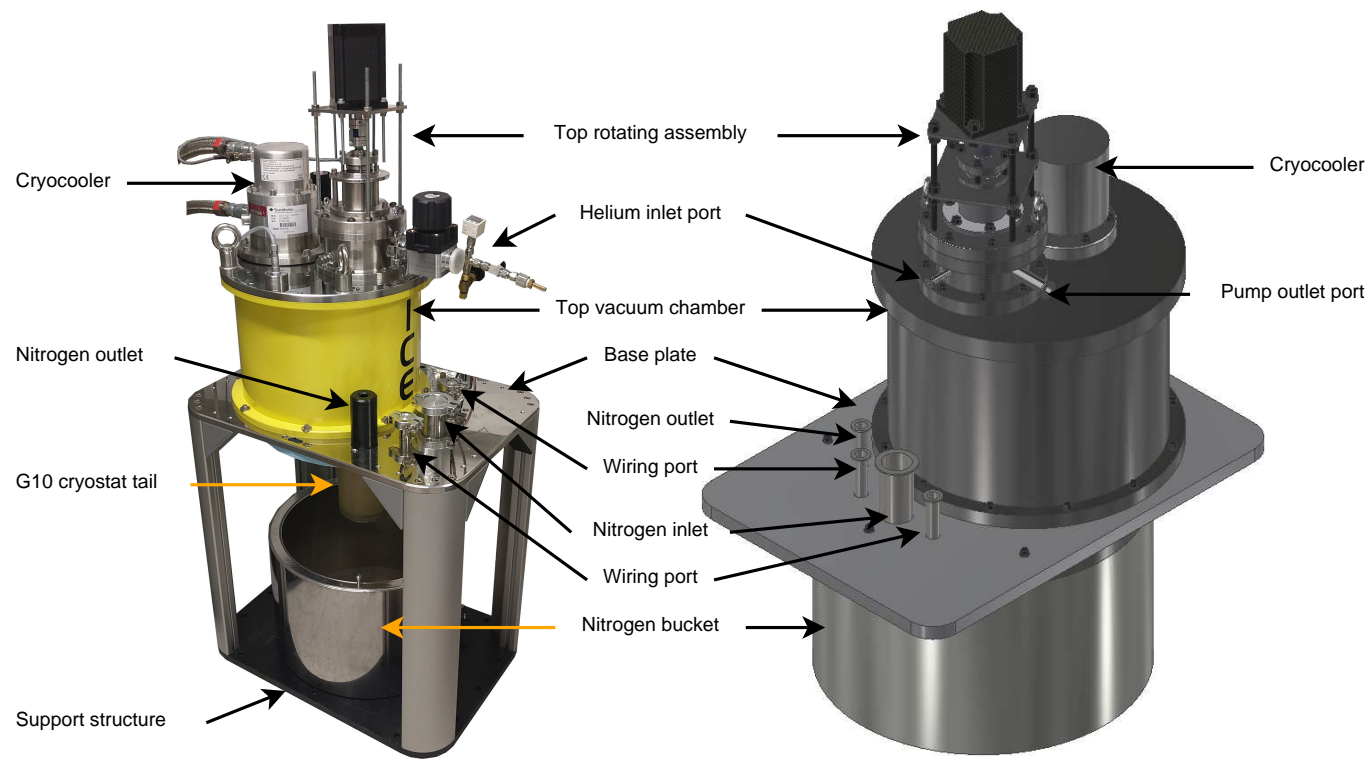

Figure 2: External view of the system with labeled connectors.

elements of the system.

Below the flange, radiation screens made of polished steel are connected by studdings. The screens are separated by spacers made of extruded polypropylene. The spacers are supposed to minimize the flow of gas to the top of the system, thus decreasing heat transfer due to convection. The bottom part of the shaft supports the rotor. It has apertures for the screw and a groove keeping the elements in proper position.

A small bearing is fastened at the bottom of the G10 cryostat carrying radial roads induced during the operation of the rotor. The grease originally lubricating the bearing is replaced with graphite to allow for the operation in cryogenic temperatures. Its small size allows to accommodate the rotor as low in the cryostat as possible. This allows a larger stator to be placed inside the nitrogen bucket.

\section{Electromagnetic design and measurement system}

The view of the system with a stator installed is shown in figure 5a. The stator is 2-pole with 3-phase distributed winding. It is expected that the application of distributed winding will reduce demagnetisation due to lower gradients present in the generated magnetic induction. The rotor consists of 


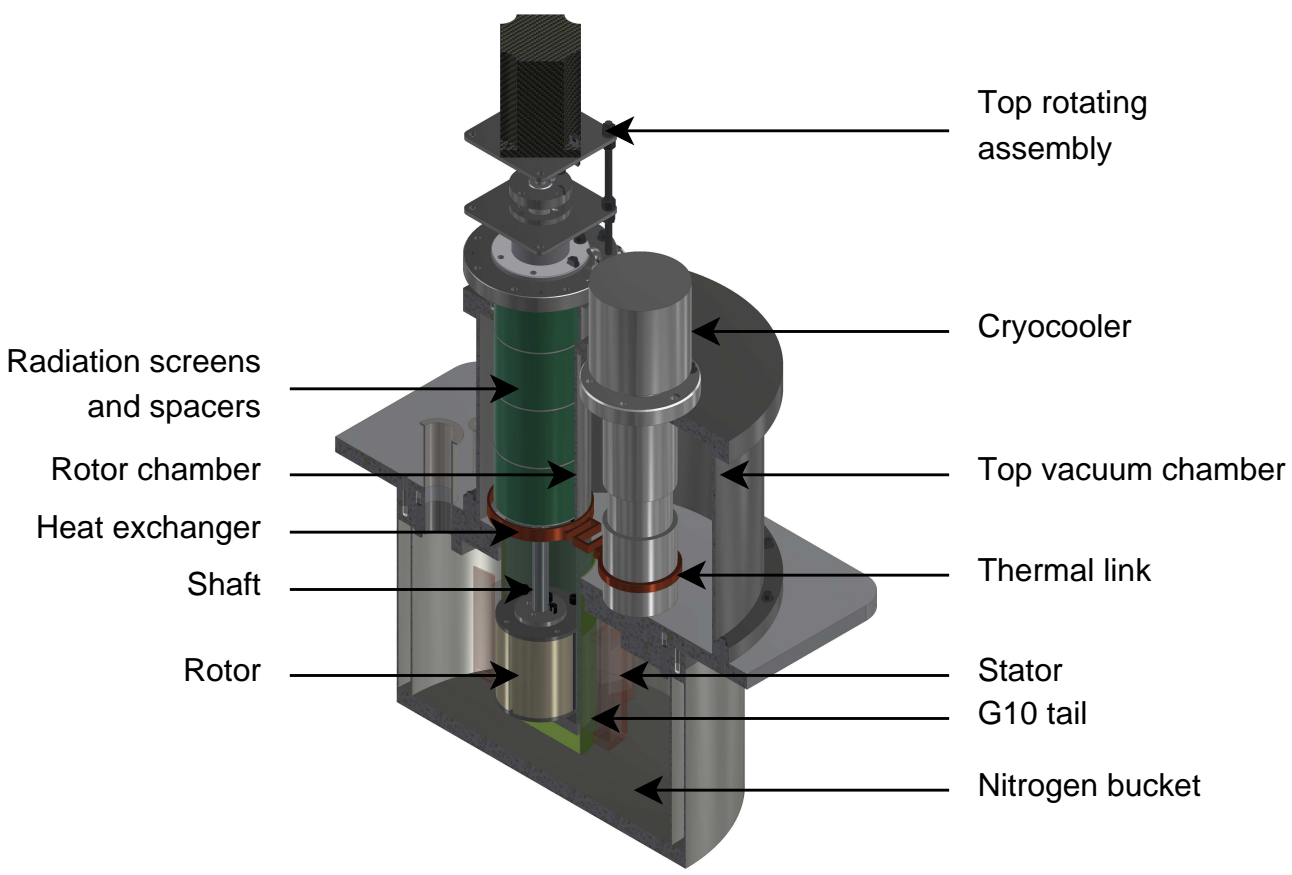

Figure 3: Cross-section of the system. Most important components are labeled.

four laminated petals. The petals are separated by 5 -mm wide rectangular slits into which the superconducting stacks are placed. Then the rotor is wrapped with tape to prevent the stacks from falling out during the operation. The stacks can be replaced to analyze the behaviour of trapped flux with different architecture and geometry.

The overview of the power and measurement circuitry is presented in figure 6 . The stator is powered by a large capacitor bank used to both magnetise and demagnetise the rotor. The capacitor bank allows to obtain short pulses of electric current of approximately 700 A used for magnetisation. During demagnetisation the rotor is rotated by the stepper motor and the stator coils can be powered to generate cross-fields. The capacitor bank is controlled by a computer with custom-written software.

During measurements the rotor with stacks is run by a RS 1417 stepper motor driven by the Geckodrive G201X drive. It is steered by employing an Agilent 33220A waveform generator. The motor allows to achieve rotational speed of approximately $600 \mathrm{rpm}$. In further development of the setup, an 


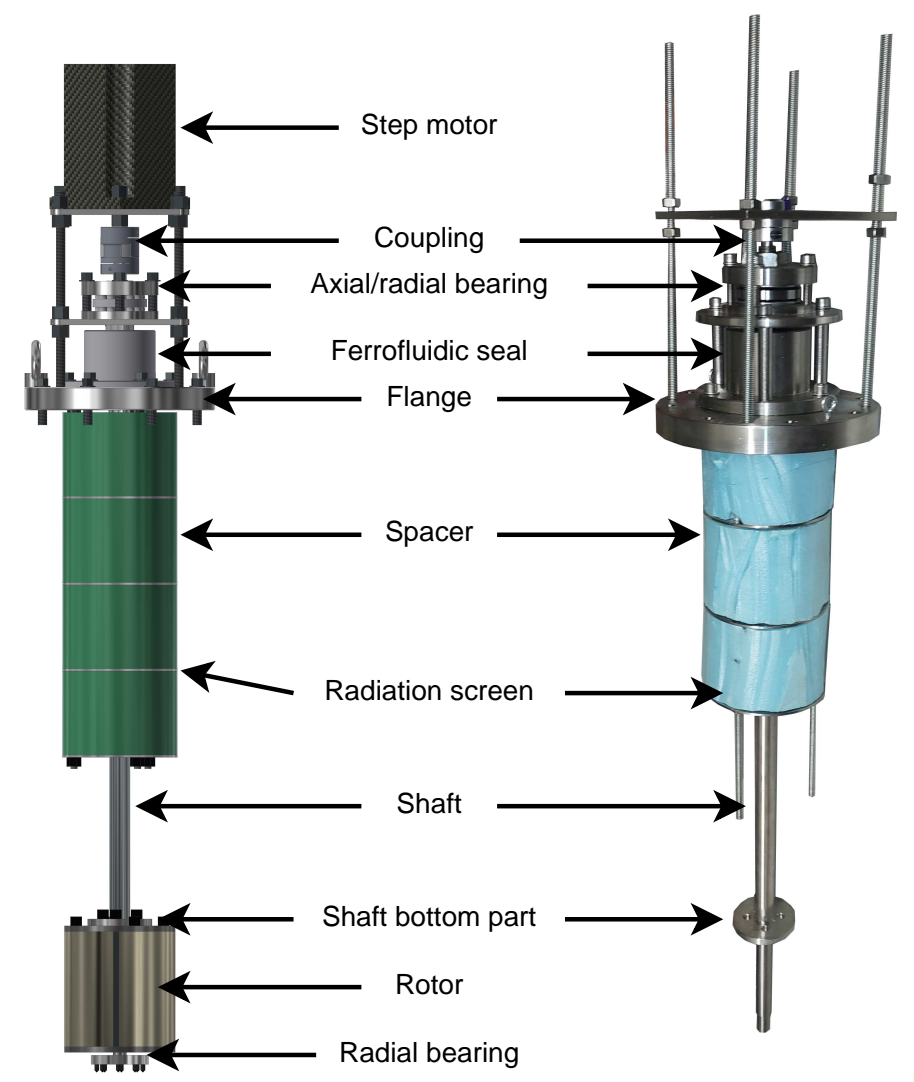

Figure 4: Insert with rotating elements of the system. The photography to the right presents the insert during assembly, the rotor and the step motor are not yet installed. The diagram to the left shows the fully assembled insert.

increase to more than $3000 \mathrm{rpm}$ is expected when using stronger motors and power sources.

A photo of the full setup in its present configuration, assembled in the laboratory, is shown in figure 7. Initial pulses from the capacitor are measured with a Fluke i1000s current probe. After a pulse, the rotor is rotated and voltage is induced in the stator coils. Both the induced voltage and pulse strength are read by a Yokogawa DL850E data recorder. Two resistance temperature sensors are placed in a system, one on the cold head of the cryocooler and the other next to the heat exchanger inside G10 cryostat. The values of temperature from both sensors are read by a Lakeshore 366 temperature controller. 


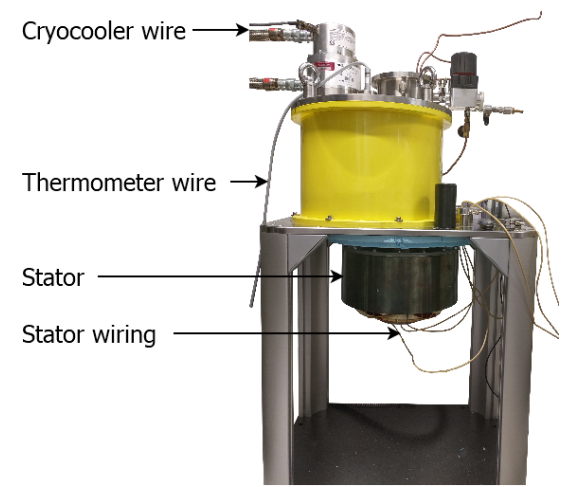

(a)

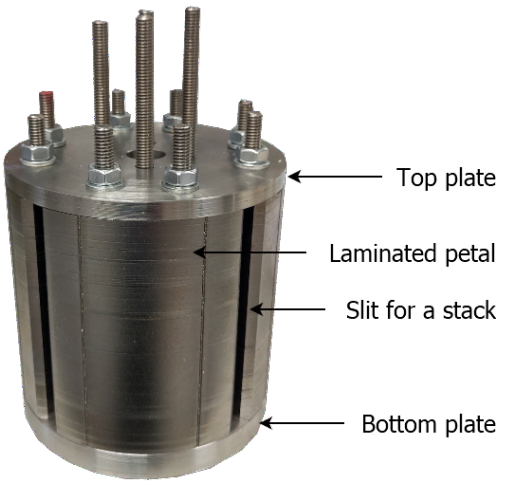

(b)

Figure 5: View of the stator (a) and the rotor (b) used in the system. The most important elements are labeled.

\section{Operation of the system}

Before starting the operation of the system, the magnetic axis of the stator, dependent on its geometry is determined. A series of relatively weak pulses moves the rotor into stable position. A dipole field is produced by the pulses while the coils of the stator are connected, hence during the magnetisation of the stator, the position of stacks with respect to magnetic field is known.

During the initial phase of operation, the top vacuum chamber and the rotor chamber are evacuated. The top vacuum chamber, serving as an insulation, is actively evacuated during the whole operation of the system. The Pfeiffer Vacuum HiPace 80 turbopump allows to obtain and maintain vacuum conditions in the pressures as low as $1 \cdot 10^{-7}$ bar for the present system. The rotor chamber is flushed and then filled with helium until a pressure of 1.5 bar at normal temperature is reached. During flushing, a DVP LC4 vacuum pump was used.

When the rotor chamber is filled with helium and vacuum insulation is in steady-state, cool-down is initiated. The setup was cooled over the span of several hours. The final temperature in a rotor chamber heat exchanger typically reaches $16 \mathrm{~K}$ but may slightly vary depending on vacuum and heat leaks. The cooling power of the used cryocooler is approximately $16 \mathrm{~W}$ at this temperature, allowing to estimate heat leaks at this level. This temperature is close to the actual temperature of a hydrogen-cooled motor in a hydrogen-fueled aircraft. 


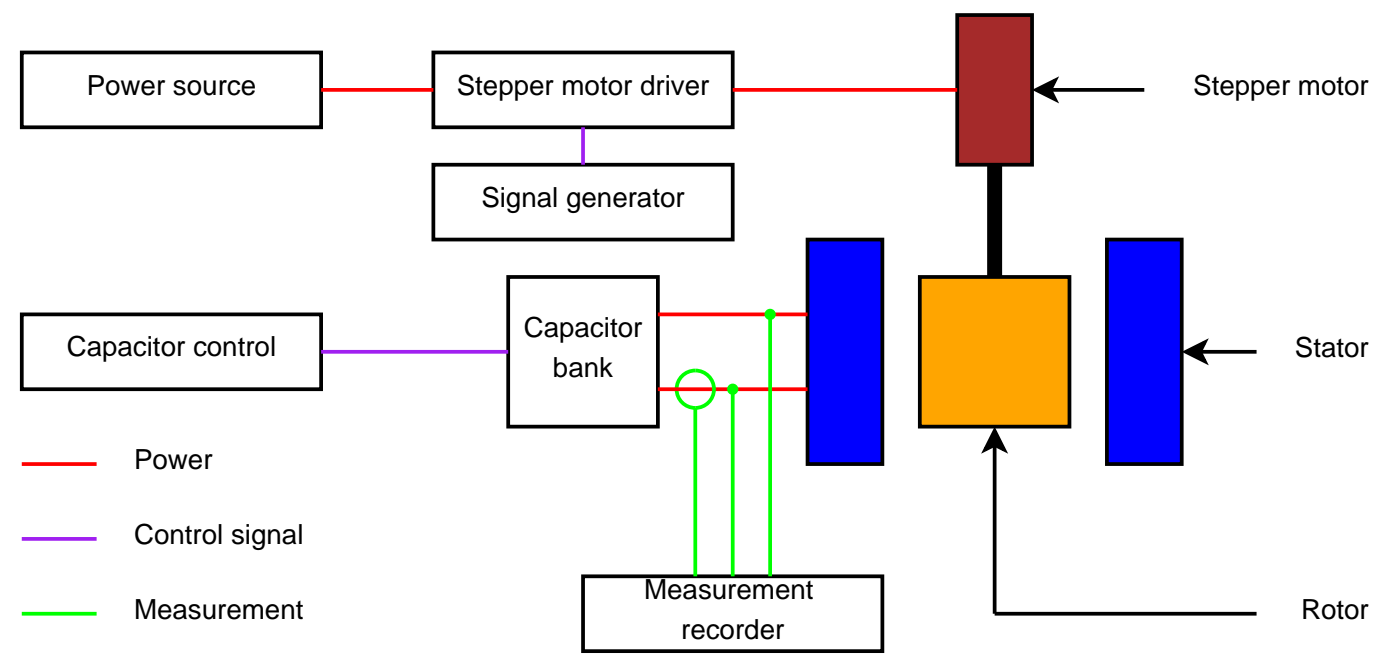

Figure 6: General diagram of the electrical connections in the system.

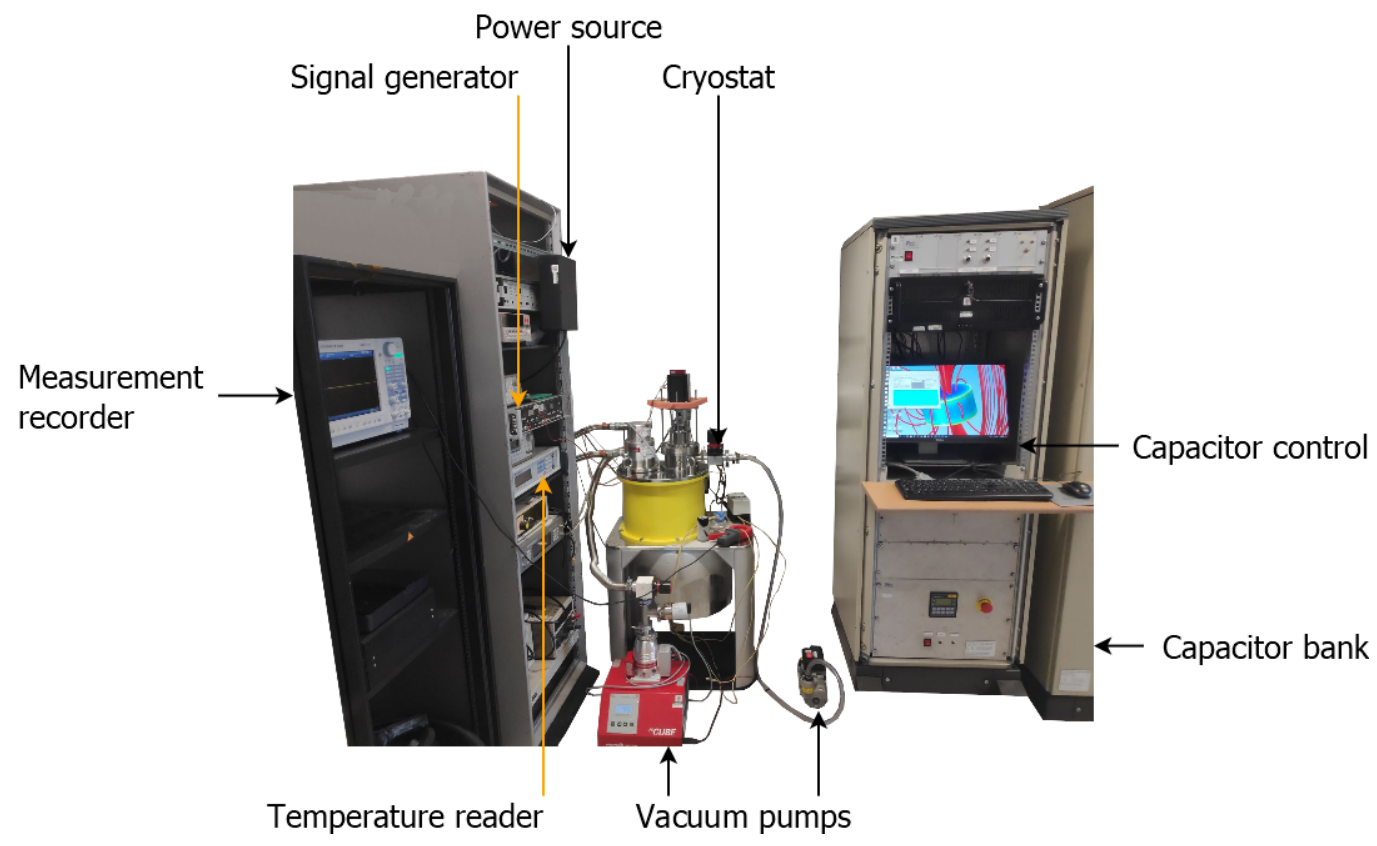

Figure 7: Full system in the laboratory.

After the rotor is cooled, it is magnetised by a pulse of magnetic flux generated by the stator connected in the same configuration as during the search for the magnetic axis. An example of the current passed through the stator is 
shown in figure 8a. The maximum pulse current used during measurements was 600 A. No damage was observed while pulsing, nor while cooling the stator with liquid nitrogen .

The magnetised rotor is then rotated by stepper motor and the voltage induced in the stator coils is read. The angular velocity of the rotor is determined based on the output from stepper motor and further confirmed by the analysis of an obtained waveform. Due to the shape of the magnetising field, only two out of the total four different stacks are fully magnetised. The stacks include a standard stack made of impregnated superconducting tapes, an interlayered stack with ferromagnetic layers, a shielded stack and a sectioned stack [49]. In order to perform analysis of all of them, at least two measuring cycles have to be performed. Results obtained during the first run of the motor are shown in figure $8 \mathrm{~b}$.

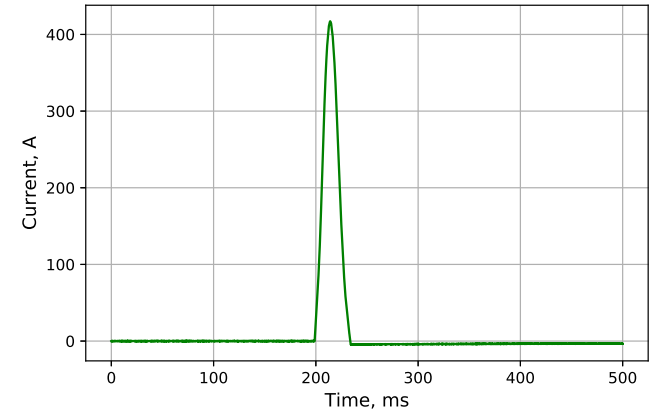

(a)

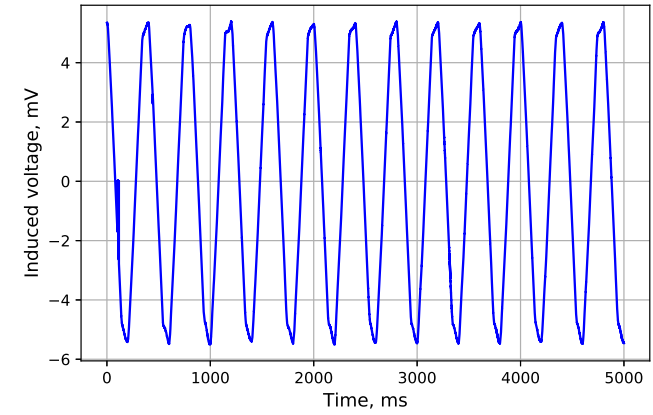

(b)

Figure 8: Example of (a) a magnetising pulse, (b) values of induced voltage obtained during the first run of the system.

Heat generation inside of stack is very small with such test conditions. There is no external magnetic field applied and most of energy dissipation is connected with induction of voltage at the coils of the stator. The operational temperature of the stacks in the rotor is thus assumed to be the same as the measured temperature of the rotor chamber. Demagnetisation tests with the application of cross-fields are planned, where heat generation will be significantly higher and can affect the superconducting properties of the stacks. 


\section{Conclusions}

Future fully electric planes will require a reliable and light source of mechanical power. A fully superconducting motor may offer a very high power density. However, such machines still suffer from issues such as demagnetisation and degradation of the trapped flux magnets. Before being widely applied, a solution for stopping this process needs to emerge.

The system presented in this paper provides a platform for experimental characterisation of the operation of stacks of different architecture and materials in the context of operation of a motor. It should be noted that when designing such a setup, one has to foresee multiple challenges, such as providing the tightness for a rotating assembly and obtaining low heat leaks allowing for the operation at low temperatures.

Future aircrafts will greatly benefit from the synergies provided by the presence of liquid hydrogen on-board. It can be used as a light and clean fuel, that can be directly converted to electric power. Additionally, its usage as coolant for the motor promotes the usage of light superconducting machines. As such, it is a real alternative to conventional solutions based on batteries and normal electric motors. Other applications can also greatly benefit from this approach. An example can be a wind turbine connected to a hydrogenbased energy storage system or a fully electric ship.

Further experiments using the setup are expected to bring valuable data, which will allow to design and build a practical superconducting motor. They are aimed at better understanding of the behaviour of the stacks in the conditions reflecting the operation of an actual motor, in terms of temperature and rotational velocity. This initial test validates the system as an appropriate tool to perform experiments leading to the improvement of the technology of trapped-flux magnets. It can be operated for prolonged periods of time, allowing one to study the demagnetisation of stacks over large number of cycles. Moreover, since the stator is capable of generating cross-fields, a solution to the demagnetisation problem can be ultimately found. Finally, with

a proper current source, the normal operation of the fully-superconducting motor can be studied.

\section{Acknowledgements}

This research is financially supported partially by the European Union's Horizon 2020 research innovation programme under grant agreement No. 
7231119 (ASuMED "Advanced Superconducting Motor Experimental Demonstrator") and also by EPSRC grant No. EP/P000738/1 entitled "Development of superconducting composite permanent magnets for synchronous motors: an enabling technology for future electric aircraft".

The authors wish to thank Dr Algirdas Baskys and Dr Anup Patel for their contributions towards the design of the system.

The authors wish to thank Oswald Elektromotoren GmbH for the manufacturing and the delivery of the stator and the elements of the rotor.

\section{References}

[1] J. Rohacs, D. Rohacs, Energy coefficients for comparison of aircraft supported by different propulsion systems, Energy 191 (2020) 116391. doi:https://doi.org/10.1016/j.energy.2019.116391.

URL http://www.sciencedirect.com/science/article/pii/S0360544219320869

[2] O. Keysan, D. Olczak, M. A. Mueller, A modular superconducting generator for offshore wind turbines, Journal of Superconductivity and Novel Magnetism 26 (5) (2013) 2103-2108. doi:10.1007/s10948-012-1950-1.

URL https ://doi .org/10.1007/s10948-012-1950-1

[3] J. H. Kim, S. Park, T. D. Le, H. C. Jo, Y.-S. Jo, Y. H. Choi, H. Lee, H. M. Kim, Analysis of the Mechanical Characteristics of a 17-MWClass High-Temperature Superconducting Synchronous Motor, Journal of Superconductivity and Novel Magnetism 28 (2) (2015) 671-679. doi:10.1007/s10948-014-2810-y. URL https://doi.org/10.1007/s10948-014-2810-y

[4] V. Climente-Alarcon, A. Patel, A. Baskys, B. A. Glowacki, Design considerations for electric motors using stacks of high temperature superconducting tape as permanent magnets, IOP Conference Series: Materials Science and Engineering 502 (2019) 012182. doi:10.1088/1757899x/502/1/012182.

URL https://doi .org/10.1088/2F1757-899x/2F502/2F1/2F012182

[5] A. Patel, A. Baskys, T. Mitchell-Williams, A. McCaul, W. Coniglio, J. Hänisch, M. Lao, B. A. Glowacki, A trapped field of $17.7 \mathrm{~T}$ in a stack of high temperature superconducting tape, Superconductor Science and Technology 31 (9) (2018) 09LT01. doi:10.1088/1361-6668/aad34c.

URL https ://doi .org/10.1088/2F1361-6668/2Faad34c 
[6] S. Zou, V. M. R. Zermeño, A. Baskys, A. Patel, F. Grilli, B. A. Glowacki, Simulation and experiments of stacks of high temperature superconducting coated conductors magnetized by pulsed field magnetization with multi-pulse technique, Superconductor Science and Technology 30 (1) (2016) 014010. doi:10.1088/0953-2048/30/1/014010. URL https://doi.org/10.10882F0953-20482F302F12F014010

[7] J. M. Collins, D. McLarty, All-electric commercial aviation with solid oxide fuel cell-gas turbine-battery hybrids, Applied Energy 265 (2020) 114787. doi:https://doi.org/10.1016/j.apenergy.2020.114787. URL http://www. sciencedirect.com/science/article/pii/S0306261920302993

[8] V. Climente-Alarcon, A. Smara, N. Mineev, L. Tomkow, B. A. Glowacki, T. Reis, Trapped-flux magnets characterization for application in synchronous machines, Journal of Physics: Conference Series 1559 (2020) 012148. doi:10.1088/1742-6596/1559/1/012148. URL https://doi.org/10.10882F1742-65962F15592F12F012148

[9] S. Zanegin, N. Ivanov, D. Shishov, I. Shishov, K. Kovalev, V. Zubko, Manufacturing and Testing of AC HTS-2 Coil for Small Electrical Motor, Journal of Superconductivity and Novel Magnetism (Jul 2019). doi:10.1007/s10948-019-05226-1. URL https://doi.org/10.1007/s10948-019-05226-1

[10] C. Wen, J. Liu, Z. Yu, J. Liu, Z. Zhao, J. Wang, Research on superconducting magnet in a superconducting synchronous generator, Journal of Superconductivity and Novel Magnetism (Apr 2019). doi:10.1007/s10948-019-5113-5.

URL https://doi.org/10.1007/s10948-019-5113-5

[11] J. Sliwinski, A. Gardi, M. Marino, R. Sabatini, Hybridelectric propulsion integration in unmanned aircraft, Energy 140 (2017) 1407 - 1416, advanced Energy Technologies in Aviation. doi:https://doi.org/10.1016/j.energy.2017.05.183.

URL http://www. sciencedirect.com/science/article/pii/S0360544217309799

[12] L. Tomkow, A. Smara, B. A. Glowacki, Application of hot press bending for shaping a stack of hts tapes operating as a trapped flux magnet, IEEE Transactions on Applied Superconductivity (2020) 11doi:10.1109/TASC.2020.2994518. 
[13] M. Park, M. Choi, S. Hahn, G. Cha, J. Lee, Effect of the stack in HTS tapes exposed to external magnetic field, IEEE Transactions on Applied Superconductivity 14 (2) (2004) 1106-1109. doi:10.1109/TASC.2004.830429.

[14] N. Bykovsky, D. Uglietti, R. Wesche, P. Bruzzone, Design Optimization of Round Strands Made by Twisted Stacks of HTS Tapes, IEEE Transactions on Applied Superconductivity 26 (2) (2016) 1-7. doi:10.1109/TASC.2016.2517187.

[15] N. Bykovsky, G. D. Marzi, D. Uglietti, P. Bruzzone, L. Muzzi, Magnetization loss for stacks of ReBCO tapes, Superconductor Science and Technology 30 (2) (2016) 024010. doi:10.1088/1361-6668/30/2/024010. URL https://doi.org/10.1088/2F1361-6668/2F30/2F2/2F024010

[16] D. Diev, A. Galimov, A. Ilin, H. Khodzhibagiyan, I. Kovalev, M. Makarenko, A. Naumov, M. Novikov, S. Novikov, A. Polyakov, V. Shcherbakov, S. Shevchenko, D. Shutova, M. Surin, HTS current leads for the NICA accelerator complex, Cryogenics 94 (2018) $45-55$. doi:https://doi.org/10.1016/j.cryogenics.2018.07.006.

URL http://www. sciencedirect.com/science/article/pii/S0011227518301553

[17] W. Goldacker, F. Grilli, E. Pardo, A. Kario, S. Schlachter, M. Vojenčiak, Roebel cables from REBCO coated conductors: a one-century-old concept for the superconductivity of the future, Superconductor Science and Technology 27 (2014) 093001.

URL https://doi.org/10.1088/0953-2048/27/9/093001

[18] T. B. Mitchell-Williams, A. Patel, A. Baskys, S. C. Hopkins, A. Kario, W. Goldacker, B. A. Glowacki, Toward uniform trapped field magnets using a stack of Roebel cable offcuts, IEEE Transactions on Applied Superconductivity 26 (3) (2016) 1-4. doi:10.1109/TASC.2016.2518994.

[19] A. Baskys, A. Patel, S. C. Hopkins, V. Kalitka, A. Molodyk, B. A. Glowacki, Self-Supporting Stacks of Commercial Superconducting Tape Trapping Fields up to $1.6 \mathrm{~T}$ Using Pulsed Field Magnetization, IEEE Transactions on Applied Superconductivity 25 (3) (2015) 1-4. doi:10.1109/TASC.2014.2360871. 
[20] L. Tomkow, A. Smara, V. Climente-Alarcon, B. A. Glowacki, Distribution of trapped magnetic flux in superconducting stacks magnetised by angled field, Journal of Superconductivity and Novel Magnetism (Dec 2019). doi:10.1007/s10948-019-05375-3.

URL https://doi.org/10.1007/s10948-019-05375-3

[21] A. Smara, N. Mineev, V. Climente-Alarcon, L. Tomkow, T. Reis, B. A. Glowacki, Influence of architecture of composite superconducting tapebased stacks on ac demagnetization for electric machines application, IEEE Transactions on Applied Superconductivity 30 (4) (2020) 1-6. doi:10.1109/TASC.2020.2990826.

[22] V. Climente-Alarcon, N. Mineev, A. Smara, L. Tomkow, B. A. Glowacki, Superconducting magnetic heterostructured components for electric motor applications, IEEE Transactions on Applied Superconductivity 30 (4) (2020) 1-5. doi:10.1109/TASC.2020.2969071.

[23] T. B. Mitchell-Williams, A. Baskys, S. C. Hopkins, V. Kalitka, A. Molodyk, B. A. Glowacki, A. Patel, Uniform trapped fields produced by stacks of HTS coated conductor tape, Superconductor Science and Technology 29 (8) (2016) 085008. doi:10.1088/0953-2048/29/8/085008. URL https://doi.org/10.1088/2F0953-2048/2F29/2F8/2F085008

[24] I. A. Rudnev, A. I. Podlivaev, Magnetic Response of the Stacks of HTS Tapes, IEEE Transactions on Applied Superconductivity 26 (4) (2016) 1-4. doi:10.1109/TASC.2016.2516347.

[25] A. Baskys, A. Patel, V. Climente-Alarcon, B. A. Glowacki, Remanent magnetic flux distribution in superconducting-ferromagnetic layered heterostructures, Journal of Superconductivity and Novel Magnetism (Feb 2019). doi:10.1007/s10948-019-5022-7.

URL https://doi.org/10.1007/s10948-019-5022-7

[26] M. Baghdadi, H. S. Ruiz, T. A. Coombs, Nature of the low magnetization decay on stacks of second generation superconducting tapes under crossed and rotating magnetic field experiments, Scientific Reports 8 (1) (2018) 1342. doi:10.1038/s41598-018-19681-8.

URL https://doi.org/10.1038/s41598-018-19681-8 
[27] V. Climente-Alarcon, N. Mineev, A. Smara, L. Tomkow, B. A. Glowacki, Superconducting magnetic heterostructured components for electric motor applications, IEEE Transactions on Applied Superconductivity (2020) 1-1doi:10.1109/TASC.2020.2969071.

[28] M. Schwarz, K.-P. Weiss, R. Heller, W. H. Fietz, Thermal conductivity measurement of hts tapes and stacks for current lead applications, Fusion Engineering and Design 84 (7) (2009) 1748 1750, proceeding of the 25th Symposium on Fusion Technology. doi:https://doi.org/10.1016/j.fusengdes.2008.11.031.

URL http://www. sciencedirect.com/science/article/pii/S0920379608003864

[29] V. Climente-Alarcon, A. Smara, L. Tomkow, B. A. Glowacki, T. Reis, Testing of surface mounted superconducting stacks as trapped-flux magnets in a synchronous machine, IEEE Transactions on Applied Superconductivity (2020) 1-1doi:10.1109/TASC.2020.2976603.

[30] J. H. Kim, C. J. Hyeon, H. L. Quach, S. H. Chae, Y. S. Chae, J. H. Moon, C.-J. Boo, Y. S. Yoon, J. Lee, H. Jeon, S. Han, Y.-G. Kim, H. Lee, H. M. Kim, Design, analysis, and fabrication of salient field-pole for a 1-kw-class hts rotating machine, Cryogenics 97 (2019) 126 - 132. doi:https://doi.org/10.1016/j.cryogenics.2018.10.018. URL http://www. sciencedirect.com/science/article/pii/S0011227518302546

[31] T. Karashima, T. Nakamura, M. Okuno, Multidisciplinary analysis of the transient performance of a $20 \mathrm{kw}$ class hts induction/synchronous motor cooled with a cryocooler and gaseous air-gap coolant, Cryogenics 99 (2019) 61-67. doi:https://doi.org/10.1016/j.cryogenics.2019.02.003. URL http://www.sciencedirect.com/science/article/pii/S001122751830300X

[32] N. Suttell, J. Vargas, J. Ordonez, S. Pamidi, C. Kim, Modeling and optimization of gaseous helium (ghe) cooled high temperature superconducting (hts) dc cables for high power density transmission, Applied Thermal Engineering 143 (2018) 922 - 934. doi:https://doi.org/10.1016/j.applthermaleng.2018.08.031. URL http://www. sciencedirect.com/science/article/pii/S1359431118313851

[33] P. Song, T.-M. Qu, L.-F. Lai, M.-S. Wu, X.-Y. Yu, Z. Han, Thermal analysis for the HTS stator consisting of HTS armature windings and an iron core for a $2.5 \mathrm{~kW}$ HTS generator, Superconductor Science and 
Technology 29 (5) (2016) 054007. doi:10.1088/0953-2048/29/5/054007.

URL https://doi.org/10.10882F0953-20482F292F52F054007

[34] L. Tomkow, N. Mineev, A. Smara, V. Climente-Alarcon, B. A. Głowacki, Theoretical analysis of heat transport in tilted stacks of HTS tapes at temperatures above $20 \mathrm{~K}$, Cryogenics 105 (2020) 103017. doi:https://doi.org/10.1016/j.cryogenics.2019.103017.

URL http://www. sciencedirect.com/science/article/pii/S0011227519302589

[35] L. Tomkow, N. Mineev, A. Smara, V. Climente-Alarcon, B. A. Glowacki, Heat extraction from HTS tape stacks applied in a superconducting motor in different cooling conditions, Journal of Physics: Conference Series 1559 (2020) 012088. doi:10.1088/1742-6596/1559/1/012088. URL https://doi.org/10.10882F1742-65962F15592F12F012088

[36] A. Campbell, M. Baghdadi, A. Patel, D. Zhou, K. Y. Huang, Y. Shi, T. Coombs, Demagnetisation by crossed fields in superconductors, Superconductor Science and Technology 30 (3) (2017) 034005. doi:10.1088/1361-6668/aa52f2.

URL https://doi.org/10.10882F1361-66682Faa52f2

[37] V. Climente-Alarcon, A. Smara, A. Patel, B. A. Glowacki, A. Baskys, T. Reis, Field cooling magnetization and losses of an improved architecture of trapped-field superconducting rotor for aircraft applications, in: AIAA Propulsion and Energy Forum and Exposition, Indianapolis, Indiana, 2019, p. 3189332.

[38] N. Bykovsky, D. Uglietti, R. Wesche, P. Bruzzone, Cyclic load effect on round strands made by twisted stacks of HTS tapes, Fusion Engineering and Design 124 (2017) 6 - 9, proceedings of the 29th Symposium on Fusion Technology (SOFT-29) Prague, Czech Republic, September 5-9, 2016. doi:https://doi.org/10.1016/j.fusengdes.2017.04.050.

URL http://www. sciencedirect.com/science/article/pii/S0920379617304532

[39] A. Smara, N. Mineev, V. Climente-Alarcon, A. Patel, A. Baskys, B. A. Glowacki, T. Reis, An experimental assessment of rotor superconducting stack demagnetization in a liquid nitrogen environment, Superconductor Science and Technology 32 (8) (2019) 085009. doi:10.1088/13616668/ab20bf.

URL https://doi.org/10.10882F1361-66682Fab20bf 
[40] A. Baskys, A. Patel, B. A. Glowacki, Measurements of crossed-field demagnetisation rate of trapped field magnets at high frequencies and below 77 k, Superconductor Science and Technology 31 (6) (2018) 065011. doi:10.1088/1361-6668/aabf32.

URL https://doi.org/10.1088/2F1361-6668/2Faabf32

[41] S. Zou, V. M. R. Zermeño, F. Grilli, Simulation of stacks of hightemperature superconducting coated conductors magnetized by pulsed field magnetization using controlled magnetic density distribution coils, IEEE Transactions on Applied Superconductivity 26 (3) (2016) 1-5. doi:10.1109/TASC.2016.2520210.

[42] T. Naito, H. Fujishiro, Y. Yamada, Thermal conductivity of single and multi-stacked DI-BSCCO , Cryogenics 49 (8) (2009) $429-432$. doi:https://doi.org/10.1016/j.cryogenics.2009.06.005.

URL http://www. sciencedirect.com/science/article/pii/S001122750900099X

[43] L. Tomkow, E. Kulikov, K. Kozlowski, V. Drobin, Improvement of the homogeneity of magnetic field by the attenuation of a selected component with an open superconducting shield made of commercial tapes, Journal of Applied Physics 126 (8) (2019) 083903. doi: $10.1063 / 1.5112036$.

URL https://doi.org/10.1063/1.5112036

[44] A. Dadhich, E. Pardo, M. Kapolka, Time constant of the transversefield demagnetization of superconducting stacks of tapes, Superconductor Science and Technology (2020).

URL http://iopscience.iop.org/10.1088/1361-6668/ab877b

[45] L. Tomkow, V. Climente-Alarcon, N. Mineev, A. Smara, B. A. Glowacki, Frequency-dependent demagnetisation rate of a shielded HTS tape stack, Journal of Physics: Conference Series 1559 (2020) 012056. doi:10.1088/1742-6596/1559/1/012056.

URL https://doi.org/10.10882F1742-65962F15592F12F012056

[46] A. Patel, V. Climente-Alarcon, A. Baskys, B. A. Glowacki, T. Reis, Design considerations for fully superconducting synchronous motors aimed at future electric aircraft (Nov 2018). doi:10.1109/ESARSITEC.2018.8607734. 
[47] M. Baghdadi, H. S. Ruiz, J. F. Fagnard, M. Zhang, W. Wang, T. A. Coombs, Investigation of demagnetization in hts stacked tapes implemented in electric machines as a result of crossed magnetic field, IEEE Transactions on Applied Superconductivity 25 (3) (2015) 1-4. doi:10.1109/TASC.2014.2372873.

[48] J. Geng, H. Zhang, C. Li, X. Zhang, B. Shen, T. A. Coombs, Angular dependence of direct current decay in a closed YBCO doublepancake coil under external AC magnetic field and reduction by magnetic shielding, Superconductor Science and Technology 30 (3) (2017) 035022. doi:10.1088/1361-6668/aa599b.

URL https://doi.org/10.10882F1361-66682Faa599b

[49] I. M. Harca, K. Machaj, L. Tomkow, B. A. Glowacki, Magnetic flux in stacks of superconducting tapes of different architecture, Superconductor Science and Technology 33 (11) (2020) 115004. doi:10.1088/13616668/abb11c.

URL https://doi.org/10.10882F1361-66682Fabb11c 